Open Access

\title{
Research as storytelling: the use of video for mixed methods research
}

\author{
Erica B. Walker ${ }^{1 *}$ (D) and D. Matthew Boyer ${ }^{2}$
}

\author{
* Correspondence: eblack4@ \\ clemson.edu \\ ${ }^{1}$ Department of Graphic \\ Communication, Clemson \\ University, 207 Godfrey Hall, \\ Clemson, SC 29634, USA \\ Full list of author information is \\ available at the end of the article
}

\begin{abstract}
Background: Mixed methods research commonly uses video as a tool for collecting data and capturing reflections from participants, but it is less common to use video as a means for disseminating results. However, video can be a powerful way to share research findings with a broad audience especially when combining the traditions of ethnography, documentary filmmaking, and storytelling.

Results: Our literature review focused on aspects relating to video within mixed methods research that applied to the perspective presented within this paper: the history, affordances and constraints of using video in research, the application of video within mixed methods design, and the traditions of research as storytelling. We constructed a Mind Map of the current literature to reveal convergent and divergent themes and found that current research focuses on four main properties in regards to video: video as a tool for storytelling/research, properties of the camera/video itself, how video impacts the person/researcher, and methods by which the researcher/viewer consumes video. Through this process, we found that little has been written about how video could be used as a vehicle to present findings of a study.

From this contextual framework and through examples from our own research, we present current and potential roles of video storytelling in mixed methods research. With digital technologies, video can be used within the context of research not only as data and a tool for analysis, but also to present findings and results in an engaging way.

Conclusions: In conclusion, previous research has focused on using video as a tool for data collection and analysis, but there are emerging opportunities for video to play an increased role in mixed methods research as a tool for the presentation of findings. By leveraging storytelling techniques used in documentary film, while staying true to the analytical methods of the research design, researchers can use video to effectively communicate implications of their work to an audience beyond academics and use video storytelling to disseminate findings to the public.

Keywords: Video, Mixed methods, Storytelling, Video research
\end{abstract}

\section{Background}

Using motion pictures to support ethnographic research began in the late nineteenth century when both fields were early in their development (Henley, 2010; "Using Film in Ethnographic Field Research, - The University of Manchester," n.d). While technologies have changed dramatically since the 1890s, researchers are still employing visual media to support social science research. Photographic imagery and video footage can be integral aspects of data collection, analysis, and reporting research studies. As

(c) The Author(s). 2018 Open Access This article is distributed under the terms of the Creative Commons Attribution 4.0 International License (http://creativecommons.org/licenses/by/4.0/), which permits unrestricted use, distribution, and reproduction in any medium, provided you give appropriate credit to the original author(s) and the source, provide a link to the Creative Commons license, and indicate if changes were made. 
digital cameras have improved in quality, size, and affordability, digital video has become an increasingly useful tool for researchers to gather data, aid in analysis, and present results.

Storytelling, however, has been around much longer than either video or ethnographic research. Using narrative devices to convey a message visually was a staple in the theater of early civilizations and remains an effective tool for engaging an audience today. Within the medium of video, storytelling techniques are an essential part of a documentary filmmaker's craft. Storytelling can also be a means for researchers to document and present their findings. In addition, multimedia outputs allow for interactions beyond traditional, static text (R. Goldman, 2007; Tobin \& Hsueh, 2007). Digital video as a vehicle to share research findings builds on the affordances of film, ethnography, and storytelling to create new avenues for communicating research (Heath, Hindmarsh, \& Luff, 2010).

In this study, we look at the current literature regarding the use of video in research and explore how digital video affordances can be applied in the collection and analysis of quantitative and qualitative human subject data. We also investigate how video storytelling can be used for presenting research results. This creates a frame for how data collection and analysis can be crafted to maximize the potential use of video data to create an audiovisual narrative as part of the final deliverables from a study. As researchers we ask the question: have we leveraged the use of video to communicate our work to its fullest potential? By understanding the role of video storytelling, we consider additional ways that video can be used to not only collect and analyze data, but also to present research findings to a broader audience through engaging video storytelling. The intent of this study is to develop a frame that improves our understanding of the theoretical foundations and practical applications of using video in data collection, analysis, and the presentation of research findings.

\section{Literature review}

The review of relevant literature includes important aspects for situating this exploration of video research methods: the history, affordances and constraints of using video in research, the use of video in mixed methods design, and the traditions of research as storytelling. Although this overview provides an extensive foundation for understanding video research methods, this is not intended to serve as a meta-analysis of all publications related to video and research methods. Examples of prior work provide a conceptual and operational context for the role of video in mixed methods research and present theoretical and practical insights for engaging in similar studies. Within this context, we examine ethical and logistical/procedural concerns that arise in the design and application of video research methods, as well as the affordances and constraints of integrating video. In the following sections, the frame provided by the literature is used to view practical examples of research using video.

The history of using video in research is founded first in photography and next in film followed more recently, by digital video. All three tools provide the ability to create instant artifacts of a moment or period of time. These artifacts become data that can be analyzed at a later date, perhaps in a different place and by a different audience, giving researchers the chance to intricately and repeatedly examine the archive of information 
contained within. These records "enable access to the fine details of conduct and interaction that are unavailable to more traditional social science methods" (Heath et al., 2010, p. 2).

In social science research, video has been used for a range of purposes and accompanies research observation in many situations. For example, in classroom research, video is used to record a teacher in practice and then used as a guide and prompt to interview the teacher as they reflect upon their practice (e.g. Tobin \& Hsueh, 2007). Video captures events from a situated perspective, providing a record that "resists, at least in the first instance, reduction to categories or codes, and thus preserves the original record for repeated scrutiny" (Heath et al., 2010, p. 6). In analysis, these audio-visual recordings allow the social science researcher the chance to reflect on their subjectivities throughout analysis and use the video as a microscope that "allow(s) actions to be observed in a detail not even accessible to the actors themselves" (Knoblauch \& Tuma, 2011, p. 417).

Examining the affordances and constraints of video in research provides a researcher the opportunity to examine the value of including video within a study. An affordance of video, when used in research, is that it allows the researcher to see an event through the camera lens either actively or passively and later share what they have seen, or more specifically, the way they saw it (Chalfen, 2011). Cameras can be used to capture an event in three different modes: Responsive, Interactive, and Constructive. Responsive mode is reactive. In this mode, the researcher captures and shows the viewer what is going on in front of the lens but does not directly interfere with the participants or events. Interactive mode puts the filmmaker into the storyline as a participant and allows the viewer to observe the interactions between the researcher and participant. One example of video captured in Interactive mode is an interview. In Constructive mode, the researcher reprocesses the recorded events to create an explicitly interpretive final product through the process of editing the video (MacDougall, 2011). All of these modes, in some way, frame or constrain what is captured and consequently shared with the audience.

Due to the complexity of the classroom-research setting, everything that happens during a study cannot be captured using video, observation, or any other medium. Video footage, like observation, is necessarily selective and has been stripped of the full context of the events, but it does provide a more stable tool for reflection than the ever-changing memories of the researcher and participants (Roth, 2007). Decisions regarding inclusion and exclusion are made by the researcher throughout the entire research process from the initial framing of the footage to the final edit of the video. Members of the research team should acknowledge how personal bias impacts these decisions and make their choices clear in the research protocol to ensure inclusivity (Miller \& Zhou, 2007).

One affordance of video research is that analysis of footage can actually disrupt the initial assumptions of a study. Analysis of video can be standardized or even mechanized by seeking out predetermined codes, but it can also disclose the subjective by revealing the meaning behind actions and not just the actions themselves (S. Goldman \& McDermott, 2007; Knoblauch \& Tuma, 2011). However, when using subjective analysis the researcher needs to keep in mind that the footage only reveals parts of an event. Ideally, a research team has a member who acts as both a researcher and a filmmaker. 
That team member can provide an important link between the full context of the event and the narrower viewpoint revealed through the captured footage during the analysis phase.

Although many participants are initially camera-shy, they often find enjoyment from participating in a study that includes video (Tobin \& Hsueh, 2007). Video research provides an opportunity for participants to observe themselves and even share their experience with others through viewing and sharing the videos. With increased accessibility of video content online and the ease of sharing videos digitally, it is vital from an ethical and moral perspective that participants understand the study release forms and how their image and words might continue to be used and disseminated for years after the study is completed.

Including video in a research study creates both affordances and constraints regarding the dissemination of results. Finding a journal for a video-based study can be difficult. Traditional journals rely heavily on static text and graphics, but newly-created media journals include rich and engaging data such as video and interactive, web-based visualizations (Heath et al., 2010). In addition, videos can provide opportunities for research results to reach a broader audience outside of the traditional research audience through online channels such as YouTube and Vimeo.

Use of mixed methods with video data collection and analysis can complement the design-based, iterative nature of research that includes human participants. Design-based video research allows for both qualitative and quantitative collection and analysis of data throughout the project, as various events are encapsulated for specific examination as well as analyzed comparatively for changes over time. Design research, in general, provides the structure for implementing work in practice and iterative refinement of design towards achieving research goals (Collins, Joseph, \& Bielaczyc, 2004). Using an integrated mixed method design that cycles through qualitative and quantitative analyses as the project progresses gives researchers the opportunity to observe trends and patterns in qualitative data and quantitative frequencies as each round of analysis informs additional insights (Gliner et al., 2009). This integrated use also provides a structure for evaluating project fidelity in an ongoing basis through a range of data points and findings from analyses that are consistent across the project. The ability to revise procedures for data collection, systematic analysis, and presenting work does not change the data being collected, but gives researchers the opportunity to optimize procedural aspects throughout the process.

Research as storytelling refers to the narrative traditions that underpin the use of video methods to analyze in a chronological context and present findings in a story-like timeline. These traditions are evident in ethnographic research methods that journal lived experiences through a period of time and in portraiture methods that use both aesthetic and scientific language to construct a portrait (Barone \& Eisner, 2012; Heider, 2009; Lawrence-Lightfoot, 2005; Lenette, Cox \& Brough, 2013).

In existing research, there is also attention given to the use of film and video documentaries as sources of data (e.g. Chattoo \& Das, 2014; Warmington, van Gorp \& Grosvenor, 2011), however, our discussion here focuses on using media to capture information and communicate resulting narratives for research purposes. In our work, we promote a perspective on emergent storytelling that develops from data collection and analysis, allowing the research to drive the narrative, and situating it in the context 
from where data was collected. We rely on theories and practices of research and storytelling that leverage the affordances of participant observation and interview for the construction of narratives (Bailey \& Tilley, 2002; de Carteret, 2008; de Jager, Fogarty \& Tewson, 2017; Gallagher, 2011; Hancox, 2017; LeBaron, Jarzabkowski, Pratt \& Fetzer, 2017; Lewis, 2011; Meadows, 2003).

The type of storytelling used with research is distinctly different from methods used with documentaries, primarily with the distinction that, while documentary filmmakers can edit their film to a predetermined narrative, research storytelling requires that the data be analyzed and reported within a different set of ethical standards (Dahlstrom, 2014; Koehler, 2012; Nichols, 2010). Although documentary and research storytelling use a similar audiovisual medium, creating a story for research purposes is ethically-bounded by expectations in social science communities for being trustworthy in reporting and analyzing data, especially related to human subjects. Given that researchers using video may not know what footage will be useful for future storytelling, they may need to design their data collection methods to allow for an abundance of video data, which can impact analysis timelines as well. We believe it important to note these differences in the construction of related types of stories to make overt the essential need for research to consider not only analysis but also creation of the reporting narrative when designing and implementing data collection methods.

\section{Methods}

This study uses existing literature as a frame for understanding and implementing video research methods, then employs this frame as perspective on our own work, illuminating issues related to the use of video in research. In particular, we focus on using video research storytelling techniques to design, implement, and communicate the findings of a research study, providing examples from Dr. Erica Walker's professional experience as a documentary filmmaker as well as evidence from current and former academic studies. The intent is to improve understanding of the theoretical foundations and practical applications for video research methods and better define how those apply to the construction of story-based video output of research findings.

The study began with a systematic analysis of theories and practices, using interpretive analytic methods, with thematic coding of evidence for conceptual and operational aspects of designing and implementing video research methods. From this information, a frame was constructed that includes foundational aspects of using digital video in research as well as the practical aspects of using video to create narratives with the intent of presenting research findings. We used this frame to interpret aspects of our own video research, identifying evidence that exemplifies aspects of the frame we used.

A primary goal for the analysis of existing literature was to focus on evidentiary data that could provide examples that illuminate the concepts that underpin the understanding of how, when, and why video research methods are useful for a range of publishing and dissemination of transferable knowledge from research. This emphasis on communicating results in both theoretical and practical ways highlighted areas within the analysis for potential contextual similarities between our work and other projects. A central reason for interpreting findings and connecting them with evidence was the need to provide examples that could serve as potentially transferable findings for others using video with their research. Given the need for a fertile environment (Zhao \& 
Frank, 2003) and attention to contextual differences to avoid lethal mutations (Brown \& Campione, 1996), understand that these examples may not work for every situation, but the intent is to provide clear evidence of how video research methods can leverage storytelling to report research findings in a way that is consumable by a broader audience.

In the following section, we present findings from the review of research and practice, along with evidence from our work with video research, connecting the conceptual and operational frame to examples and teasing out aspects from existing literature.

\section{Results and findings}

When looking at the current literature regarding the use of video in research, we developed a Mind Map to categorize convergent and divergent themes in the current literature, see Fig. 1. Although this is far from a complete meta-analysis on video research (notably absent is a comprehensive discussion of ethical concerns regarding video research), the Mind Map focuses on four main properties in regards to video: video as a tool for storytelling/research, properties of the camera/video itself, how video impacts the person/researcher, and methods by which the researcher/viewer consumes video.

Video, when used as a tool for research, can document and share ethnographic, epistemic, and storytelling data to participants and to the research team (R. Goldman, 2007; Heath et al., 2010; Miller \& Zhou, 2007; Tobin \& Hsueh, 2007). Much of the research in this area focuses on the properties (both positive and negative) inherent in the camera itself such as how video footage can increase the ability to see and experience the world, but can also act as a selective lens that separates an event from its natural context (S. Goldman \& McDermott, 2007; Jewitt, n.d.; Knoblauch \& Tuma, 2011; MacDougall, 2011; Miller \& Zhou, 2007; Roth, 2007; Sossi, 2013).

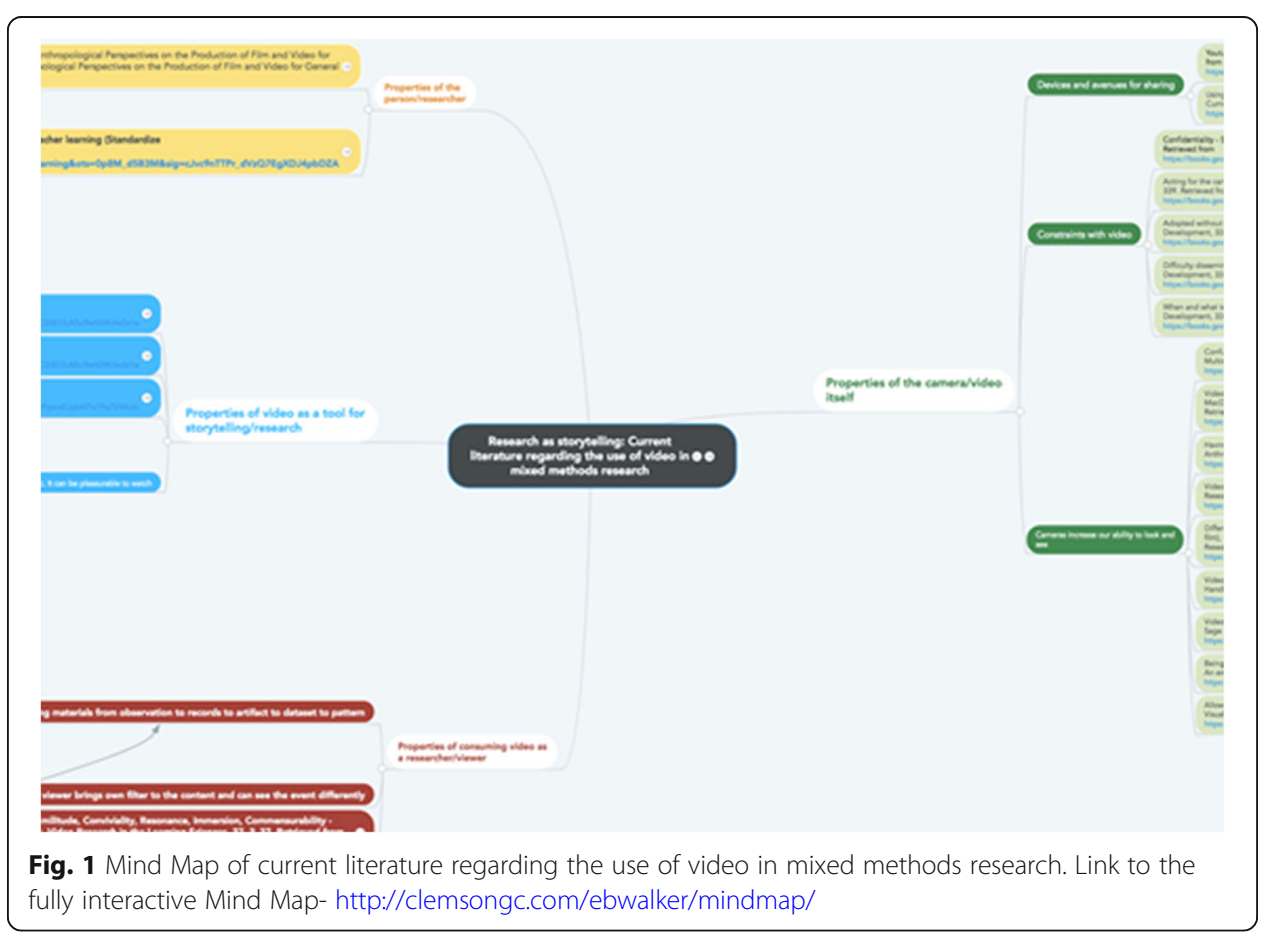


Some research speaks to the role of the video-researcher within the context of the study, likening a video researcher to a participant-observer in ethnographic research (Derry, 2007; Roth, 2007; Sossi, 2013). The final category of research within the Mind Map focuses on the process of converting the video from an observation to records to artifact to dataset to pattern (Barron, 2007; R. Goldman, 2007; Knoblauch \& Tuma, 2011; Newbury, 2011). Through this process of conversion, the video footage itself becomes an integral part of both the data and findings.

The focus throughout current literature was on video as data and the role it plays in collection and analysis during a study, but little has been written about how video could be used as a vehicle to present findings of a study. Current literature also did not address whether video-data could be used as a tool to communicate the findings of the research to a broader audience.

In a recent two-year study, the research team led by Dr. Erica Walker collected several types of video footage with the embedded intent to use video as both data and for telling the story of the study and findings once concluded (Walker, 2016). The study focused on a multidisciplinary team that converted a higher education Engineering course from lecture-based to game-based learning using the Cognitive Apprenticeship educational framework. The research questions examined the impact that the intervention had on student learning of domain content and twenty-first Century Skills. Utilizing video as both a data source and a delivery method was built into the methodology from the beginning. Therefore, interviews were conducted with the researchers and instructors before, during, and after the study to document consistency and changes in thoughts and observations as the study progressed. At the conclusion of the study, student participants reflected on their experience directly through individual video interviews. In addition, every class was documented using two static cameras, placed at different angles and framing, and a mobile camera unit to capture closeup shots of student-instructor, student-student, and student-content interactions. This resulted in more than six-hundred minutes of interview footage and over five-thousand minutes of classroom footage collected for the study.

Video data can be analyzed through quantitative methods (frequencies and word maps) as well as qualitative methods (emergent coding and commonalities versus outliers). Ideally, both methods are used in tandem so that preliminary results can continue to inform the overall analysis as it progresses. In order to capitalize on both methods, each interview was transcribed. The researchers leveraged digital and analog methods of coding such as digital word-search alongside hand coding the printed transcripts. Transcriptions contained timecode notations throughout, so coded segments could quickly be located in the footage and added to a timeline creating preliminary edits.

There are many software workflows that allow researchers to code, notate timecode for analysis, and pre-edit footage. In the study, Opportunities for Innovation: Game-based Learning in an Engineering Senior Design Course, NVivo qualitative analysis software was used together with paper-based analog coding. In a current study, also based on a higher education curriculum intervention, we are digitally coding and pre-trimming the footage in Adobe Prelude in addition to analog coding on the printed transcripts. Both workflows offer advantages. NVivo has built-in tools to create frequency maps and export graphs and charts relevant to qualitative analysis whereas Adobe Prelude adds coding notes directly into the footage metadata and connects directly with Adobe Premiere video editing software, which streamlines the editing process. 
From our experience with both workflows, Prelude works better for a research team that has multiple team members with more video experience because it aligns with video industry workflows, implements tools that filmmakers already use, and Adobe Team Projects allows for co-editing and coding from multiple off-site locations. On the other hand, NVivo works better for research teams where members have more separate roles. NVivo is a common qualitative-analysis software so team members more familiar with traditional qualitative research can focus on coding and those more familiar with video editing can edit based on those codes allowing each team member to work within more familiar software workflows.

In both of these studies, assessments regarding storytelling occurred in conjunction with data processing and analysis. As findings were revealed, appropriate clips were grouped into timelines and edited to produce a library of short, topic-driven videos posted online, see Fig. 2. A collection of story-based, topic-driven videos can provide other practitioners and researchers a first-hand account of how a study was designed and conducted, what worked well, recommendations of what to do differently, participant perspectives, study findings, and suggestions for further research. In fact, the videos cover many of the same topics traditionally found in publications, but in a collection of short videos accessible to a broad audience online.

By sharing the results of the study publicly online, conversations between practitioners and researchers can develop on a public stage. Research videos are easy to share across social media channels which can broaden the academic audience and potentially open doors for future research collaborations. As more journals move to accept multi-media studies,

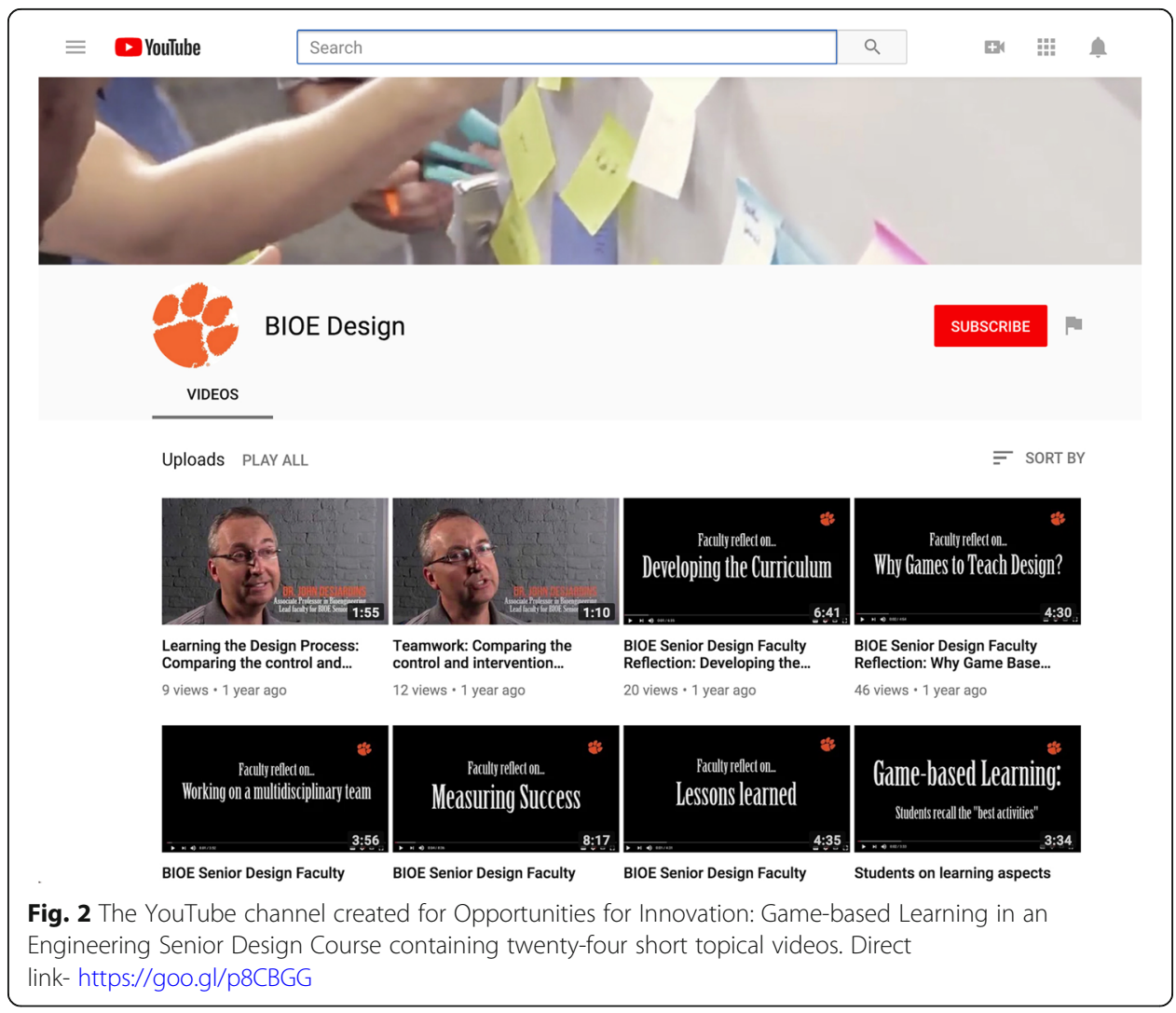


publicly posted videos provide additional ways to expose both academics and the general public to important study results and create easy access to related resources.

\section{Discussion}

Video research as storytelling: The intersection and divergence of documentary filmmaking and video research

"Film and writing are such different modes of communication, filmmaking is not just a way of communicating the same kinds of knowledge that can be conveyed by an anthropological text. It is a way of creating different knowledge" (MacDougall, 2011).

When presenting research, choosing either mode of communication comes with affordances and constraints for the researcher, the participants, and the potential audience.

Many elements of documentary filmmaking, but not all, are relevant and appropriate when applied to gathering data and presenting results in video research. Documentary filmmakers have a specific angle on a story that they want to share with a broad audience. In many cases, they hope to incite action in viewers as a response to the story that unfolds on screen. In order to further their message, documentarians carefully consider the camera shots and interview clips that will convey the story clearly in a similar way to filmmakers in narrative genres. Decisions regarding what to capture and how to use the footage happen throughout the entire filmmaking process: prior to shooting footage (pre-production), while capturing footage (production), and during the editing phase (post-production).

Video researchers can employ many of the same technical skills from documentary filmmaking including interview techniques such as pre-written questions; camera skills such as framing, exposure, and lighting; and editing techniques that help draw a viewer through the storyline (Erickson, 2007; Tobin \& Hsueh, 2007). In both documentary filmmaking and in video research, informed decisions are made about what footage to capture and how to employ editing techniques to produce a compelling final video.

Where video research diverges from documentary filmmaking is in how the researcher thinks about, captures, and processes the footage. Video researchers collect video as data in a more exploratory way whereas documentary filmmakers often look to capture preconceived video that will enable them to tell a specific story. For a documentary filmmaker, certain shots and interview responses are immediately discarded as they do not fit the intended narrative. For video researchers, all the video that is captured throughout a study is data and potentially part of the final research narrative. It is during the editing process (post-production) where the distinction between data and narrative becomes clear.

During post-production, video researchers are looking for clips that clearly reflect the emergent storylines seen in the collective data pool rather than the footage necessary to tell a predetermined story. Emergent storylines can be identified in several ways. Researchers look for divergent statements (where an interview subject makes unique observation different from other interviewees), convergent statements (where many different interviewees respond similarly), and unexpected statements (where something different from what was expected is revealed) (Knoblauch \& Tuma, 2011). 
When used thoughtfully, video research provides many sources of rich data. Examples include reflections of the experience, in the direct words of participants, that contain insights provided by body language and tone, an immersive glimpse into the research world as it unfolds, and the potential to capture footage throughout the entire research process rather than just during prescribed times. Video research becomes especially powerful when combined with qualitative and quantitative data from other sources because it can help reveal the context surrounding insights discovered during analysis.

We are not suggesting that video researchers should become documentary filmmakers, but researchers can learn from the stylistic approaches employed in documentary filmmaking. Video researchers implementing these tools can leverage the strengths of short-format video as a storytelling device to share findings with a more diverse audience, increase audience understanding and consumption of findings, and encourage a broader conversation around the research findings.

\section{Implications for future work}

As the development of digital media technologies continues to progress, we can expect new functionalities far exceeding current tools. These advancements will continue to expand opportunities for creating and sharing stories through video. By considering the role of video from the first stages of designing a study, researchers can employ methods that capitalize on these emerging technologies. Although they are still rapidly advancing, researchers can look for ways that augmented reality and virtual reality could change data analysis and reporting of research findings. Another emergent area is the use of machine learning and artificial intelligence to rapidly process video footage based on automated thematic coding. Continued advancements in this area could enable researchers to quickly quantify data points in large quantities of footage.

In addition to exploring new functionalities, researchers can still use current tools more effectively for capturing data, supporting analysis, and reporting findings. Mobile devices provide ready access to collect periodic video reflections from study participants and even create research vlogs (video blogs) to document and share ongoing studies as they progress. In addition, participant-created videos are rich artifacts for evaluating technical and conceptual knowledge as well as affective responses. Most importantly, as a community, researchers, designers, and documentarians can continue to take strengths from each field to further the reach of important research findings into the public sphere.

\section{Conclusions}

In conclusion, current research is focused on using video as a tool for data collection and analysis, but there are new, emerging opportunities for video to play an increased and diversified role in mixed methods research, especially as a tool for the presentation and consumption of findings. By leveraging the storytelling techniques used in documentary filmmaking, while staying true to the analytical methods of research design, researchers can use video to effectively communicate implications of their work to an 
audience beyond academia and leverage video storytelling to disseminate findings to the public.

Funding

There was no external or internal funding for this study.

\section{Availability of data and materials}

Data is available in the Mind Map online which visually combines and interprets the full reference section available at the end of the full document.

\section{Authors' contributions}

Article was co-written by both authors and based on previous work by Dr. Walker where Dr. Boyer served in the role of dissertation Committee Chair. Both authors read and approved the final manuscript.

\section{Competing interests}

Neither of the authors have any competing interest regarding this study or publication.

\section{Publisher's Note}

Springer Nature remains neutral with regard to jurisdictional claims in published maps and institutional affiliations.

\section{Author details}

${ }^{1}$ Department of Graphic Communication, Clemson University, 207 Godfrey Hall, Clemson, SC 29634, USA. ${ }^{2}$ College of Education, Clemson University, 207 Tillman Hall, Clemson, SC 29634, USA.

Received: 10 May 2018 Accepted: 4 July 2018

Published online: 28 July 2018

\section{References}

Bailey, P. H., \& Tilley, S. (2002). Storytelling and the interpretation of meaning in qualitative research. J Adv Nurs, 38(6), 574-583. http://doi.org/10.1046/j.1365-2648.2000.02224.x

Barone, T., \& Eisner, E. W. (2012). Arts based research (pp. 1-183). https://doi.org/10.4135/9781452230627

Barron B (2007) Video as a tool to advance understanding of learning and development in peer, family, and other informal learning contexts. Video Research in the Learning Sciences:159-187

Brown AL, Campione JC (1996) Psychological theory and the design of innovative learning environments: on procedures, principles and systems. In: Schauble L, Glaser R (eds) Innovations in learning: new environments for education. Lawrence Erlbaum Associates, Hillsdale, NJ, pp 234-265

Chalfen, R. (2011). Looking Two Ways: Mapping the Social Scientific Study of Visual Culture. In E. Margolis \& L. Pauwels (Eds.), The Sage handbook of visual research methods. books.google.com

Chattoo, C. B., \& Das, A. (2014). Assessing the Social Impact of Issues-Focused Documentaries: Research Methods and Future Considerations Center for Media \& Social Impact, 24. Retrieved from https://www.namac.org/wpcontent/ uploads/2015/01/assessing_impact_social_issue_documentaries_cmsi.pdf

Collins, A., Joseph, D., \& Bielaczyc, K. (2004). Design research: theoretical and methodological issues. Journal of the Learning Sciences, 13(1), 15-42. https://doi.org/https://doi.org/10.1207/s15327809jls1301_2

Dahlstrom, M. F. (2014). Using narratives and storytelling to communicate science with nonexpert audiences. Proc Natl Acad Sci, 111(Supplement 4), 13614-13620. http://doi.org/10.1073/pnas.1320645111

de Carteret, P. (2008). Storytelling as research praxis, and conversations that enabled it to emerge. Int J Qual Stud Educ, 21(3), 235-249. http://doi.org/10.1080/09518390801998296

de Jager A, Fogarty A, Tewson A (2017) Digital storytelling in research: a systematic review. Qual Rep 22(10):2548-2582

Derry SJ (2007) Video research in classroom and teacher learning (Standardize that!). Video Research in the Learning Sciences:305-320

Erickson F (2007) Ways of seeing video: toward a phenomenology of viewing minimally edited footage. Video Research in the Learning Sciences:145-155

Gallagher, K. M. (2011). In search of a theoretical basis for storytelling in education research: story as method. International Journal of Research and Method in Education, 34(1), 49-61. http://doi.org/10.1080/1743727X.2011. 552308

Gliner, J. A., Morgan, G. A., \& Leech, N. L. (2009). Research Methods in Applied Settings: An Integrated Approach to Design and Analysis, Second Edition. Taylor \& Francis

Goldman R (2007) Video representations and the perspectivity framework: epistemology, ethnography, evaluation, and ethics. Video Research in the Learning Sciences 37:3-37

Goldman S, McDermott R (2007) Staying the course with video analysis Video Research in the Learning Sciences:101113

Hancox, D. (2017). From subject to collaborator: transmedia storytelling and social research. Convergence, 23(1), 49-60. http://doi.org/10.1177/1354856516675252

Heath, C., Hindmarsh, J., \& Luff, P.(2010). Video in Qualitative Research. SAGE Publications. Retrieved from https://market. android.com/details?id=book-MtmViguNi4UC

Heider KG (2009) Ethnographic film: revised edition. University of Texas Press

Henley P (2010) The Adventure of the Real: Jean Rouch and the Craft of Ethnographic Cinema. University of Chicago Press 
Jewitt, C. (n.d). An introduction to using video for research - NCRM EPrints Repository. National Centre for Research Methods. Institute for Education, London. Retrieved from http://eprints.ncrm.ac.uk/2259/4/NCRM_workingpaper_ 0312.pdf

Knoblauch H, Tuma R (2011) Videography: An interpretative approach to video-recorded micro-social interaction. The SAGE Handbook of Visual Research Methods:414-430

Koehler D (2012) Documentary and ethnography: exploring ethical fieldwork models. Elon Journal Undergraduate Research in Communications 3(1):53-59 Retrieved from https://www.elon.edu/docs/e-web/academics/ communications/research/vol3no1/EJSpring12_Full.pdf\#page $=53 \mathrm{i}$

Lawrence-Lightfoot, S. (2005). Reflections on portraiture: a dialogue between art and science. Qualitative Inquiry: QI, 11(1), 3-15. https://doi.org/10.1177/1077800404270955

LeBaron, C., Jarzabkowski, P., Pratt, M. G., \& Fetzer, G. (2017). An introduction to video methods in organizational research. Organ Res Methods, 21(2), 109442811774564. http://doi.org/10.1177/10944281177445649

Lenette, C., Cox, L., \& Brough, M. (2013). Digital storytelling as a social work tool: learning from ethnographic research with women from refugee backgrounds. Br J Soc Work, 45(3), 988-1005. https://doi.org/10.1093/bjsw/bct184

Lewis, P. J. (2011). Storytelling as research/research as storytelling. Qual Inq, 17(6), 505-510. http://doi.org/10.1177/ 1077800411409883

(2011) Anthropological filmmaking: An empirical art. In: The sage handbook of visual research methods. MacDougall, D, pp 99-113

Meadows D (2003) Digital storytelling: research-based practice in new media. Visual Com(2):189-193

Miller K, Zhou X (2007) Learning from classroom video: what makes it compelling and what makes it hard. Video Research in the Learning Sciences:321-334

Newbury, D. (2011). Making arguments with images: Visual scholarship and academic publishing. In Eric Margolis \& (Ed.), The SAGE Handbook of Visual Research Methods. na

Nichols B (2010) Why are ethical issues central to documentary filmmaking? Introduction to Documentary, Second Edition. In: 42-66

Roth W-M (2007) Epistemic mediation: video data as filters for the objectification of teaching by teachers. In: Goldman R, Pea R, Barron B, Derry SJ (eds) Video research in the learning sciences. Lawrence Erlbaum Ass Mahwah, NJ, pp 367-382

Sossi, D. (2013). Digital Icarus? Academic Knowledge Construction and Multimodal Curriculum Development, 339

Tobin J, Hsueh Y (2007) The poetics and pleasures of video ethnography of education. Video Research in the Learning Sciences:77-92

Using Film in Ethnographic Field Research - Methods@Manchester - The University of Manchester. (n.d.). Retrieved March 12, 2018, from https://www.methods.manchester.ac.uk/themes/ethnographic-methods/ethnographic-fieldresearch/

Walker, E. B. (2016). Opportunities for Innovation: Game-based Learning in an Engineering Senior Design Course (PhD). Clemson University. Retrieved from http://tigerprints.clemson.edu/all_dissertations/1805/

Warmington, P., van Gorp, A., \& Grosvenor, I. (2011). Education in motion: uses of documentary film in educational research. Paedagog Hist, 47(4), 457-472. https://doi.org/10.1080/00309230.2011.588239

Zhao, Y., \& Frank, K. A. (2003). Factors affecting technology uses in schools: an ecological perspective. Am Educ Res J, 40(4), 807-840. https://doi.org/10.3102/00028312040004807

Submit your manuscript to a SpringerOpen ${ }^{\circ}$ journal and benefit from:

- Convenient online submission

- Rigorous peer review

- Open access: articles freely available online

- High visibility within the field

Retaining the copyright to your article

Submit your next manuscript at $>$ springeropen.com 